\title{
The Plasma Environment of Venus, Mars, and Titan, an Introduction
}

\author{
Karoly Szego • Ari-Matti Harri • Michel Blanc
}

Published online: 1 November 2011

(C) Springer Science+Business Media B.V. 2011

In December 2009 the EUROPLANET Research Infrastructure, a project funded by the European Community under the Capacities programme of the Seventh Framework Programme (grant agreement number 228319) and the International Space Science Institute, organized a workshop in Bern to overview and summarize our current knowledge on the plasma environments of Venus, Mars, and Titan. This was the first workshop organized in the series of four planned in the networking activity NA-2 of Europlanet RI. Emphasis was on new results, since many excellent reviews were published recently. The comparison of the plasma environment of these three solar system objects was a primary focus of the workshop, which also aimed at defining directions for future research, through further use of existing data or development of new space missions.

In this introductory paper, we summarize the main results of this workshop, via an overview of the different chapters it contains.

Unlike the Earth, our neighbours Venus and Mars have no global magnetic fields, although Mars has small-scale crustal fields; and Titan, to our best knowledge, is also a nonmagnetic body. These three objects are the only non-magnetic solar system bodies having a dense atmosphere. Venus and Mars have the supersonic and super-Alfvenic solar wind as their plasma environment. Titan, in contrast, is located inside Saturn's hot, rapidly rotating, subsonic, multicomponent magnetospheric flow (although it occasionally makes excursions into the magnetosheath) and its environment is highly variable in time. Accordingly,

K. Szego (凶)

KFKI Research Institute for Particle and Nuclear Research, Budapest, Hungary

e-mail: szego@rmki.kfki.hu

A.-M. Harri

Finnish Meteorological Institute, Helsinki, Finnland

M. Blanc

Ecole Polytechnique, Palaiseau and IRAP, Toulouse, France

M. Blanc

International Space Science Institute, Bern, Switzerland 
these objects provide a wide parameter range for the investigations of the interaction of non-magnetized objects with their plasma environments. It is remarkable that their global structure is very similar, even if each obstacle has its own characteristics depending on the actual plasma regime.

In the last few years, European and international spacecraft have gathered significant new data on the interaction of these non-magnetized objects with their plasma environments. Cassini-Huygens ${ }^{1}$ has accumulated data over 70 flybys of Titan; Mars Express ${ }^{2}$ has over seven years of data from Mars, and Venus Express ${ }^{3}$ over 4 years of data from Venus. This data bonanza will continue to accumulate during the extended phase of all of these missions, providing an ideal observational support to summarize, compare and analyse the similarities and differences of their plasma interaction. Cometary interaction with the solar wind also bears similarities with these objects; therefore the knowledge presented in this volume will be highly useful for the ROSETTA comet chaser (a mission of the European Space Agency) examining at close distance how a frozen comet is transformed by the heat of the Sun in the solar wind.

The basic processes of space plasma physics have been established by the scientific investigations during the past few decades. Currently the research is focused on the understanding of the evolution of planetary atmospheres and its effect on the climate evolution over geological time scales. That requires the study of the flow of energy and momentum from the solar wind through the different planetary plasma regions leading to the loss of planetary material; mutatis mutandis, the same holds for Titan.

Around non-magnetic solar system bodies the interaction of flowing plasma with an ionised obstacle creates a layered, onion-like structure in the plasma flow in front of the obstacle, and an elongated plasma formation behind it for typical interplanetary magnetic field (IMF) configurations. In front of the obstacle the magnetic field piles up forming a magnetic barrier. The magnetic field configuration behind the obstacle, in the tail, depends on the dynamic pressure of the flow. Recent investigations have revealed that when the IMF is parallel to the flow, a more complex plasma structure is generated around the obstacle, and the magnetic barrier is missing. The features of the interaction, including the nature and the features of the boundary layers, can be very different, depending upon the properties of the incident plasma and the nature of the obstacle. A particular role is played by the pickup processes. The pickup ion radius depends on the strength of the magnetic field which e.g. in the case of Titan is of the same order as the obstacle radius. The outer limit of the spatial volume which is under the control of ions originating from the obstacle is the boundary of the induced magnetosphere. The boundaries around the obstacles are of finite width. This is particularly important for the cometary bow shock, its considerable width allows neutrals to be ionized within the bow shock resulting in a new category of shocks, the mass loaded shock. The mass loading region is particularly extended around Titan, its width is a few Titan radii. As a consequence, the magnetic field and the pickup velocity are continuously changing in this region. For the non-magnetized planets the induced magnetosphere is not axially symmetric, due to the electric field induced by the magnetic field and flow velocity; this introduces asymmetry also in the structure of the tail.

\footnotetext{
${ }^{1}$ The Cassini-Huygens space mission is an international cooperative effort of NASA, the European Space Agency (ESA), the Italian Space Agency (ASI) and several separate European academic and industrial contributors.

${ }^{2}$ The Mars Express space mission is a cooperative effort of the European Space Agency.

${ }^{3}$ The Venus Express space mission is a cooperative effort of the European Space Agency.
} 
The physical processes evidently involve electrons, which by definition account for half of the plasma population (except when multiply-charged ions are present). The electrons come from sources including the incoming plasma flow and the ionization processes (photo ionization and impact ionization) in the ionosphere of the object; they may also be produced in the region of the object by additional processes. The ionospheres are produced by solar radiation and precipitating electron impact (from the magnetosphere or shocked solar wind), or a combination of both. Electrons in the environment near non-magnetized objects can provide a source of energy for the atmosphere.

The papers of this volume describe first the upstream conditions, then the obstacles, the formation of the induced magnetospheres, ion acceleration and outflow; the special features derived from the observations of energetic neutrals, and a paper on modelling complement the description. Let us say a few words about each of them.

The paper by Delva et al. reviews the proton upstream waves reported for Venus and Mars, their generation mechanism and consequences for the planetary exospheres. Arridge et al. discuss the upstream parameters controlling the interaction of Titan and its effects on the chemistry of the ionosphere. The physics of Saturn's magnetosphere near Titan is also considered in this respect resulting in the high variability of the plasma environment. Titan has the most significant atmosphere of any moon in the solar system. The paper of Coates et al. reviews several recent advances in our description and understanding of Titan's ionosphere, and compares it to the ionospheres of Venus and Mars. The role of ionospheric photoelectrons is also considered. Dubinin et al. focus on the different channels through which planetary matter escapes. Forces responsible for energization in the various channels are special. The solar wind forcing of ion escape for Venus and Mars is the focal topic of the review paper of Lundin. The aim of this overview is to analyse the different acceleration processes associated with ion outflow. A broad description of the structure and properties of the induced magnetospheres and the boundaries of Venus, Mars an Titan is presented by Bertucci et al. The concept of fossil magnetic fields is also analyzed in depth. Analysis of energetic neutrals brings important information about the plasma environments and about the neutral exospheres; this novel technique is presented by Yoshifumi et al. Numerical modeling of the global plasma and neutral dynamics at Venus, Mars and Titan, presented by Kallio et al., makes this volume complete.

A focal problem of the current research is to clarify the outflow channels of the planetary plasma and the planetary plasma loss as a result of the interaction with the impinging plasma flow. A part of the outflow takes place through the tail region; another part escapes through the boundary layer of the induced magnetosphere. The driver of the outflow is different in the different channels, this is also to be clarified in the future. The sophisticated instrumentation e.g. onboard of Mars-Express and Venus-Express makes it possible to measure the tail escape velocities. Presently the scientific community is debating on how the ionospheric outflow is made possible, and what is the role of thermal escape relative to the non-thermal escape processes. This requires the categorisation of the ionospheric structure, dynamics and chemistry. Outflow in the boundaries requires the identification of the boundaries and of the role of the different frictional effects, and the processes themselves that form the boundary regions and the tail. Additional work is necessary to quantify the pickup processes and associated wave excitations.

In summary, this volume gathers in a comprehensive and systematic way a comparison of the different features of the similarities and differences in the plasma interactions of Venus, Mars and Titan. From this comparison, one can explore a significant fraction of the parameter space of magnetized plasma flow interactions with non magnetized objects with 
an atmosphere. Beyond the particular cases of Venus, Mars and Titan, this comparison partly addresses the comet-solar wind interaction, and the interaction of some of the exoplanets with their stellar wind.

Acknowledgements The editors here thank all the referees who reviewed the articles in this volume for their dedication to this work. Their contribution very much enhanced the value of this volume. 